\title{
Pseudo-Haptics for Rigid Tool/Soft Object Interaction Feedback in Virtual Environments
}

\author{
Min $\mathrm{Li}^{1}{ }^{*}$, Maisarah B. Ridzuan ${ }^{1}$, Sina Sareh ${ }^{1}$, Lakmal. D. Seneviratne ${ }^{1}$, Prokar Dasgupta ${ }^{2}$, and Kaspar \\ Althoefer $^{1}$ \\ 1. Department of Informatics, Kings College London, London, WC2R 2LS, UK. (e-mail: \{min.m.li; maisarah_binti.ridzuan; \\ sina.sareh; lakmal.seneviratne; k.althoefer\}@kcl.ac.uk). \\ 2. Medical Research Council (MRC) Centre for Transplantation, King's College London, King's Health Partners, Guy's Hospital, \\ LondonSE19RT, UK. (prokarurol@gmail.com)
}

\begin{abstract}
This paper proposes a novel pseudo-haptics soft object stiffness simulation technique which is a marked improvement to currently used simulation methods and an effective low-cost alternative to expensive 3-DOF haptic devices. Soft object stiffness simulation is achieved by maneuvering an indenter avatar over the surface of a virtual soft object by means of an input device, such as a mouse, a joystick, or a touch-sensitive tablet. The alterations to the indenter avatar behavior produced by the proposed technique create for the user the illusion of interaction with a hard inclusion embedded in the soft object. The proposed pseudo-haptics technique is validated with a series of experiments conducted by employing three types of 2-DOF force-sensitive haptic surfaces, including a touchpad, a tablet with an S-pen input, and a tablet with a bare finger input. It is found that both the sensitivity and the positive predictive value of hard inclusion detection can be significantly improved by $33.3 \%$ and $13.9 \%$ respectively by employing tablet computers. Using tablet computers could produce results comparable to direct hand touch in detecting hard inclusions in a soft object. The experimental results presented here confirm the potential of the proposed technique for conveying haptic information in rigid tool / soft object interaction in virtual environments.
\end{abstract}

Keywords - Haptic feedback, Pseudo-haptic feedback, Rigid tool/soft object interaction, Tumor identification.

\section{Introduction}

Pseudo-haptic methods have been used to simulate friction [1], stiffness of a spring [2], softness [3], mass [4], and texture [5]. In general, they create an illusion of haptic feedback via a visual display [1] without the need for the use of haptic devices, and can convey one-dimensional (1D), two-dimensional (2D), or even three-dimensional (3D) haptic information.

The new soft object stiffness simulation technique proposed in this paper uses pseudo-haptic feedback which is produced by employing Commercial Off-The-Shelf (COTS) devices which are compact, mobile, wireless and easy to manipulate. Soft object stiffness 3D data is acquired using a rolling/sliding indentation method [6] and then conveyed using a force-sensitive touchpad or a tablet computer. The advantage of the proposed technique over haptic devices employed in tool / soft object interaction simulations is that it combines effectiveness, portability, ease-of-use and low cost.

Conventional haptic feedback methods use impedance-based haptic devices. These devices can sense the kinematics of the haptic mechanism (position/velocity) and use the information to produce an output force. Haptic rendering deals with the computation of the contact forces between a haptic user's virtual environment and the virtual objects [7]. Classic haptic rendering algorithms for the simulation of rigid objects in a virtual environment are penalty-based, correlating repulsive forces to the penetration distance between the objects proportionally [8]. Since collision causes deformation, interactions with deformable objects require a special analysis. Relatively complex, bulky, and expensive haptic devices are currently used to sense the user's hand motion and provide force feedback of the deformed soft object.

PHANToM is one of the most widely used haptic devices (Sensable Technology Inc.) [9]. The PHANToM device series has three different configurations: Omni (6-DOF of movement and 3-DOF of haptic feedback), Desktop (6-DOF of movement and 3-DOF of haptic feedback) and Premium (6-DOF of movement and 6-DOF of haptic feedback). The weights of these devices are approximately $1.79 \mathrm{~kg}, 2.86 \mathrm{~kg}$, and $31.3 \mathrm{~kg}$ (a control console included), respectively. The Delta, Omega and Sigma haptic systems from Force Dimension Inc. [10] are based on a particular parallel mechanism concept. Both Delta. 3 and Omega. 3 are capable of performing three active translations. Omega. 6 adds three passive rotations, while Delta. 6 adds three active rotations. Omega. 7 introduces another grasping motion. The most recent Sigma. 7, uniquely, is based on a redesigned delta base; it introduces a more precision active grasping capability. These devices are about $270 \times 300 \times 350 \mathrm{~mm}$ in dimension. The mechanical structure of the Falcon haptic system from Novint Technologies, Inc. [11] is very similar to the Delta haptic device, but with a lower price. It has a size of $229 \times 229 \times 229 \mathrm{~mm}$ and weight of $2.72 \mathrm{~kg}$. An opposed-type multi-fingered haptic interface - a Haptic Interface Robot (HIRO) device developed by Kawasaki et al. [12] - was used for breast palpation simulation [13]. It consists of a

* Corresponding author. E-mail address: min.m.li@kcl.ac.uk. Phone: +44(0)2078482902 
force actuated 6-DOF arm and three fingers, with 3-DOF force output, which was upgraded to a five-fingered HIRO III device later [14]. It has a weight of about $3.8 \mathrm{~kg}$ with a $23 \mathrm{~kg}$ control apparatus (a box size of $443 \times 222 \times 464 \mathrm{~mm}$ ). The above devices need to be put on a flat surface, e.g. a desk, when they are in use. The Rutgers Master II force feedback glove [15] is a light-weight (Exoskeleton weight only $80 \mathrm{~g}$ ) device that can provide force feedback of up to $16 \mathrm{~N}$ to each finger. Nevertheless, apart from the glove itself, it has extra components such as pneumatic servovalves and air supply. Pneumatic actuators are used to apply forces to all of the fingertips except for the little finger. However, the glove limits the range of motion of the fingers because of the placement of the cylinders. Those haptic devices have been applied in training for knee palpation [15], abdominal palpation to detect liver tumors [16], prostate cancer palpation simulation [17], horse ovary palpation simulation [18], feline abdominal palpation simulation [19], palpation simulation in cardiovascular surgery [20], and haptic palpation [21]. One drawback of these haptic devices is their relatively high cost [22]. Moreover, those bulky devises need to be connected to a power supply when they are in use.

In recent years, the development of pressure-sensitive technology has improved touchpad and laptop user experience. Touchpad devices have been engineered to respond to different force levels applied by the user to the sensitive surface of the device. Compared to the aforementioned haptic devices, the pressure-sensitive touchpad and tablet computers proposed to use here are smaller, lighter (less than $1 \mathrm{~kg}$ in weight), less complex in structure, cheaper, and portable. Therefore, we propose to convey 3D haptic information using a force-sensitive touchpad or a tablet computer combined with the pseudo-haptic feedback technique.

In this paper the effectiveness of the proposed technique is evaluated by the measure of its ability to identify hard inclusions within soft objects in a simulation where 3D haptic information on rigid tool / soft object interaction is conveyed using a motion and force input device.

In Section 2.1, two types of reaction forces between the rigid tool tip and the soft objects are simulated. Sections 2.2, 2.3, 2.4 and 2.5 explain the concept and algorithm of applying pseudo-haptics to the soft object stiffness simulation and hard inclusions identification process by using a force-sensitive touchpad and force-sensitive tablet computer. Section 3 describes the process of soft object stiffness data acquisition, the validation test protocol; Section 3 presents the results of the validation studies. Conclusions are offered in Section 5.

\section{Methodology}

\subsection{Rigid Tool / Soft Object Interaction}

The soft object stiffness 3D haptic data which is used for the purposes of this paper comes from the rolling/sliding indentation method introduced by [6], [23]-[25] to detect tissue abnormalities.

The rolling indentation method works as follows:

During the superficial rolling/sliding, the indenter slides over a soft object surface. As the indenter approaches a hard inclusion embedded in a soft object an increase of lateral force $f_{x}$ is experienced. (see Fig. 1). During indenting behavior, a tool is pressed on the surface of a soft object to explore its stiffness pattern. The reaction force $f_{y}$ increases as the indentation depth increases. When the areas with hard inclusions underneath are pressed, the reaction forces are greater than those of other areas which are free from inclusions.

By using our proposed pseudo-haptic feedback technique, the lateral reaction force $f_{x}$ of rolling behavior and the normal reaction force $f_{y}$ of indenting behavior are simulated simultaneously.

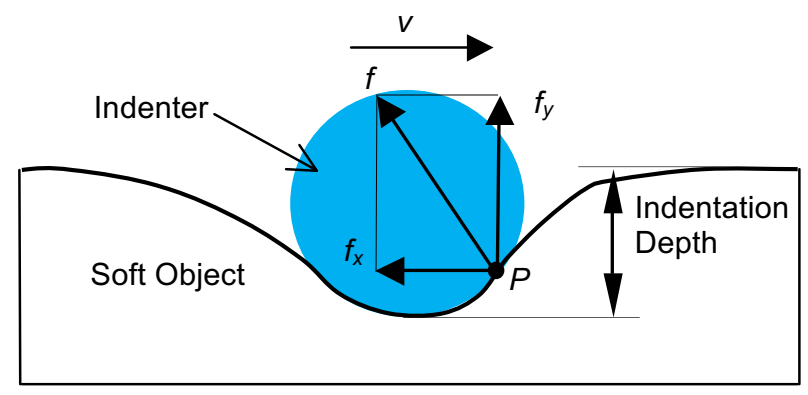

Fig. 1. Reflecting forces in rigid tool-soft object interaction.

\subsection{Pseudo-Haptic Feedback in Rigid Tool / Soft Object Interaction}

Conventional haptic feedback methods use impedance-based haptic devices which produce an output force based on the kinematics information they receive from the haptic mechanism. The position sensors embedded inside the device track and record the position data as a human user moves the end-effecter of the haptic device. As shown in Fig. 2, the hand motion $(D)$ is considered as an input signal. The relationship between the input device movement and the movement of the slave indenter avatar (i.e. the cursor) can be defined using an avatar display ratio. The avatar display ratio is the relationship between the indenter avatar displacement $(d)$ and the input device displacement $(D)(R=d / D)$. When the contact between the avatar and the soft tissue occurs, the reaction forces are calculated using the computer models of the virtual objects and the penetrated depth. The torque commands 
are sent to the actuators on the haptic interface in real time based on the calculated results. Thus, appropriate feedback reaction forces $(F F)$ can be applied to the user's hand, leading to haptic perception of virtual objects.

In the proposed pseudo-haptic technique a motion and force input device is used. When the user moves the input device towards a relatively stiffer area over a distance $(D)$, the indenter avatar display ratio will be updated to be lower than the original default ratio $\left(R_{m}<R_{o}, R_{m}\right.$ is the modified ratio and $R_{o}$ is the original default ratio). Thus, the modified avatar displacement $d_{m}$ will be lower than the original default indenter avatar displacement $d_{o}\left(d_{m}=R_{m} \cdot D, d_{o}=R_{o} \cdot D\right)$. An illusion of resistance to motion will be experienced. In this way, the virtual forces $(V F)$ can be perceived visually along with the direction of the movement.

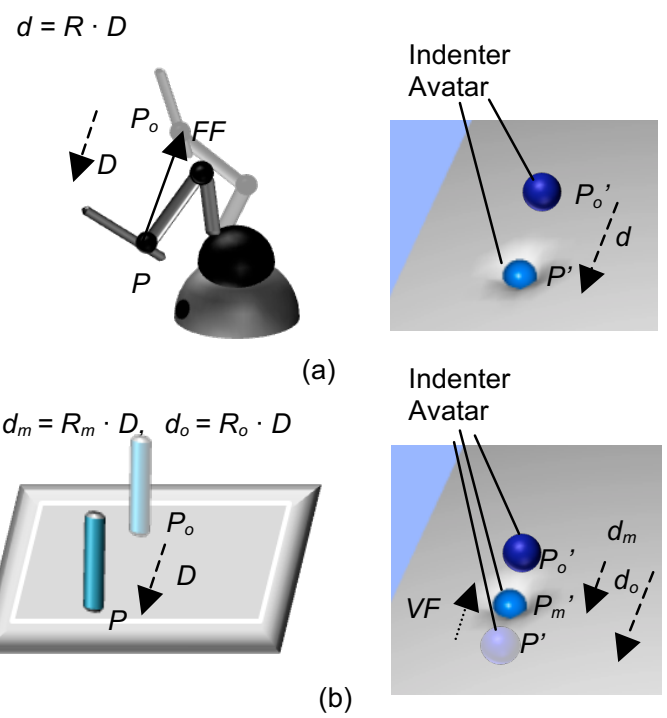

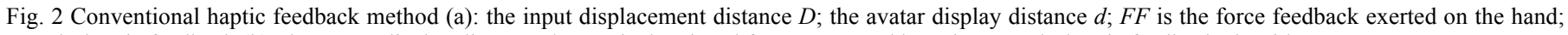
Pseudo-haptic feedback (b): the avatar display distance $d_{m} ; V F$ is the virtual force generated by using pseudo-haptic feedback algorithm.

\subsection{Pseudo-haptic soft object stiffness simulation using a force-sensitive touchpad motion input device}

Here it will be shown how 3D haptic information is conveyed using a force-sensitive Wacom BAMBOO Pen \& Touch touchpad (a 2D haptic input device, $248 \times 176 \times 8.5 \mathrm{~mm}$ in dimension, $125 \times 85 \mathrm{~mm}$ for touch sensitivity, $360 \mathrm{~g}$ weight) and our proposed pseudo-haptic feedback technique.

The flow chart of the pseudo-haptic soft object stiffness simulation using a force-sensitive touchpad motion input device is shown in Fig. 3. The user moves the pen at a tangent to the surface whilst applying a normal force on the force-sensitive touchpad. The normal force exerted on the touchpad, together with the 2-DOF movement kinematics of the pen tip over the surface of the touchpad are the two inputs of the system. The outputs are the normal reaction force from the touchpad, the virtual resistance along the movement direction generated by using pseudo-haptic feedback, and the soft object deformation shown on the graphical interface.

The force level extracted from the device is translated to a force value. Then, a stiffness value and an indentation depth are obtained from the measured stiffness distribution according to the force value and the position information. Consequently, the stiffness values and the kinematics of tangentially moving the pen around the contact point are processed in a pseudo-haptic feedback algorithm to generate the required update on the indenter avatar movement.

It should be mentioned that, here, the deformation of the soft object is calculated based on the indentation depth, the model of soft object surface deformation curvature, and the avatar movement update. The tissue curvature is calculated based on the indentation depth and the model of soft object surface deformation curvature [26]. The deformation of the soft object during interaction is displayed in real time using a geometrical deformable soft object model, which is established based on a predefined finite element model considering the indentation depth and the indenter diameter.

The details of this model are presented in [26]. As a node of the mesh is pressed by the indenter, the normal vertex of this node is redefined according to the depth of the indenter (See Figs. 4 and 5). Note that, as a result of the indentation of a specific node, the normal vertices of nodes located nearby on the mesh are also affected. If the indentation depth increases, the number of affected nodes will also increase. Thus, the pattern of the deformation of soft object is displayed graphically.

Using a force-sensitive touchpad and a special pen as an input device, the return value from the touchpad is the force level. It needs to be mapped with force magnitude. The touchpad has 1024 force levels. A Force/Torque sensor ATI Mini 40 was placed under the touchpad to record the force applied and map the force levels defined by the touchpad device to actual force values. During the experiment, the pen was used to apply normal forces to the touchpad from 0 to a maximum force level 1024 . Force levels and force values were recorded. This experiment was repeated four times. 57 sets of data points were obtained. The least square method was used to produce the regression equation described in equation $(1)\left(R^{2}=0.9703\right.$, see Fig. 6). 


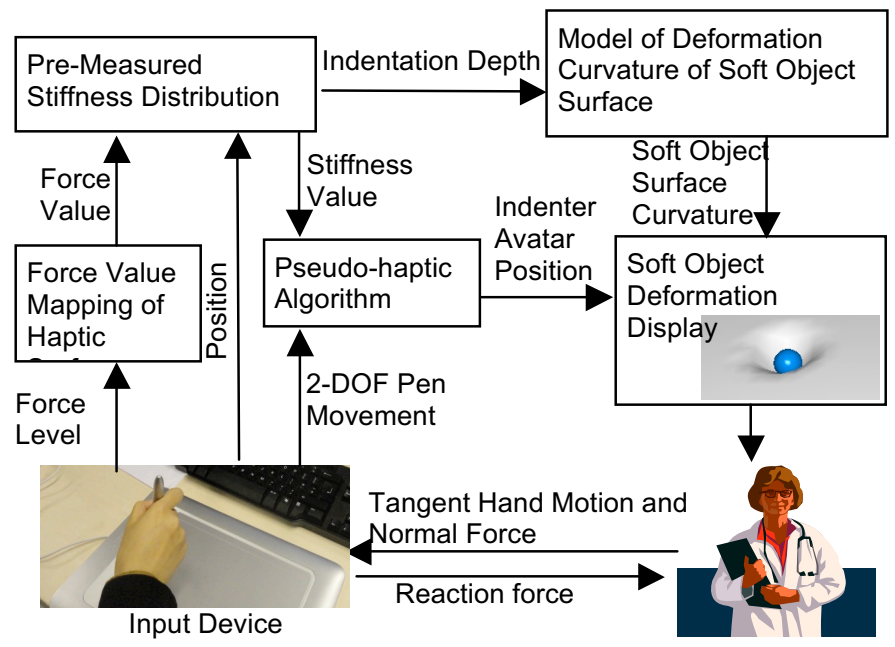

Fig. 3 The flow chart of the pseudo-haptic palpation simulation using a force-sensitive touchpad motion input device.

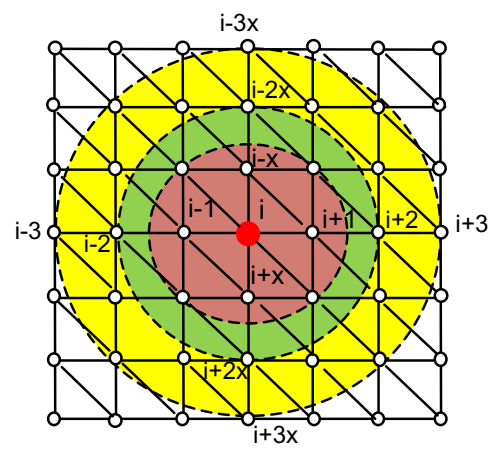

Fig. 4 The location of the indenter avatar is on node $\mathrm{i}$; the normal vertex of this node is redefined according to the depth of the indenter; the normal vertices of nodes located nearby on the mesh are also affected; as the indentation depth increases, the number of affected nodes increases.



Fig. 5 The indenter avatar (blue sphere) and the deformation of the soft object surface; as the indentation depth increases, the affected tissue surface area becomes larger.

$$
f_{n}=0.0827 e^{0.0039 f_{l}},
$$

where normal reaction force values $\left(f_{n}\right)$ is acquired from the reaction force matrices in sliding indentation [6]; $f_{l}$ is the force level reading from the touchpad data package.

The coordinates of the touchpad surface are linearly mapped to the soft object surface. The force levels $\left(f_{l}\right)$ read from the touchpad data package are converted to the normal force values $\left(f_{n}\right)$ according to the equation (1) first. The position of the interaction on the surface of the soft object is calculated based on the mapping relationship between the touchpad surface and the soft object surface. Then the indentation depth $d_{m}(z)$ is calculated as a function of the pressure applied to the touchpad $\left(f_{n}\right)$ employing a look-up table of force matrices linearly interpolated between stored values.

The tangent reaction force can be acquired via the lookup table of force matrices according to the indentation depth $d_{m}(z)$. Then, the difference of the tangent reaction forces $\left(\Delta f_{t}\right)$ between the current avatar position and the last avatar position is calculated as:

$$
\Delta f_{t}=f_{t}-f_{t l},
$$

where $f_{t l}$ is the tangent reaction force value at the current avatar position; $f_{t l}$ is the tangent reaction force value at the last avatar 
position.

When the soft object stiffness in current position is stiffer than the tissue in the previous position $\left(\Delta f_{t}>0\right)$, the movement distance is reduced:

$$
\begin{aligned}
& d_{m}(x, y)=R_{m t} \cdot D(x, y), \\
& R_{m t}=R_{o} /\left(f_{t}+1\right),
\end{aligned}
$$

where $R_{o}$ is the original default ratio between the avatar displacement and the input device displacement (avatar display ratio) calculated based on the coordinates mapping relationship between the touchpad surface and the soft object surface; and $R_{m t}$ is the modified avatar tangent display ratio.

When the soft object in current position has the same stiffness or is softer than in the previous position $\left(\Delta f_{t}<=0\right)$, the position of the indenter avatar is calculated based on the coordinates mapping relationship between the touchpad surface and the soft object surface.

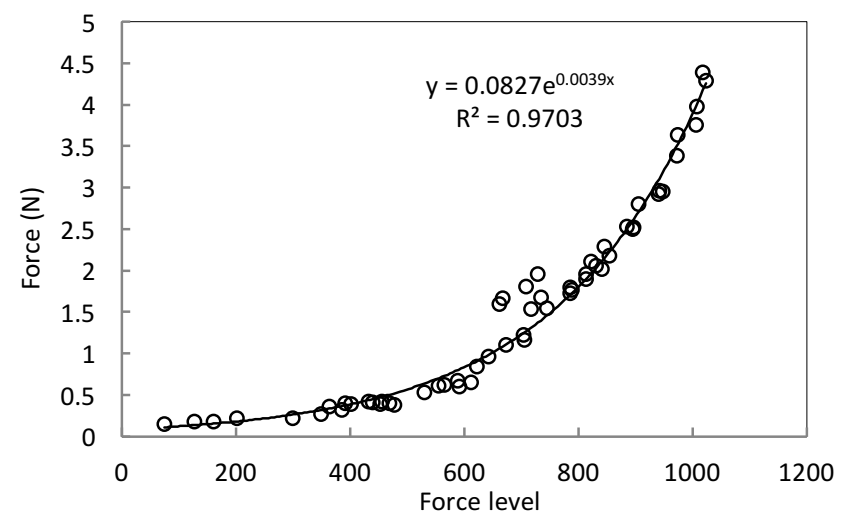

Fig. 6 Force level and force value mapping of the force-sensitive touchpad

\section{$2.4 \quad$ Pseudo-haptic soft object stiffness simulation using tablet computers}

Using a touchpad as an input device, visual and haptic information are presented at different point of interaction - the contact force is exerted from the touchpad via the special pen while the visual information displays on a computer screen. To improve the effectiveness of object stiffness identification, haptic and visual information should be presented at the same active point of interaction. Utilizing a tablet computer makes the user feel as though their finger or the stylus can penetrate the pressure-sensitive surface and be extended into the digital world to manipulate virtual tissue behind the screens directly. This is called direct touch or immersive illusion [27], [28].

Two prominent types of commercially available tablet computer are used in this study - Samsung Galaxy Note 10.1 (using an S-pen) and Motorola Xoom (using the user's bare finger). Samsung Galaxy Note 10.1 has a dimension of $262 \times 180 \times 8.9 \mathrm{~mm}$ and a weight of $600 \mathrm{~g}$. Motorola Xoom is $249 \times 167.8 \times 12.9 \mathrm{~mm}$ and $730 \mathrm{~g}$. Similar to the pen-pad interaction method explained for the touchpad device, here, the user holds the pen or uses an index finger to move tangentially over the tablet's force-sensitive surface, hence, exerting a normal force on it. These normal forces, together with the motion kinematics of the pen tip or fingertip over the surface of the tablet, are the two inputs of the system. The outputs are the normal reaction force from the tablet, the virtual resistance along the movement direction generated through pseudo-haptic feedback, and the soft object deformation shown on the graphical interface.

The Samsung Note (using a Wacom S-pen) is force-sensitive and has 1024 levels of pressure sensitivity. Motorola Xoom tablet senses the area of touch: when the touched area is broader, it recognizes the applied force as larger. Here, the force level is again mapped with force magnitude, employing the same experimental method explained in Section 2 C. This force level-force value relationship is described in equation (5) and (6) (see Figs. 7 and 8), according to which the force levels $\left(f_{l}\right)$ are converted to the normal force values $\left(f_{n}\right)$. Then the indentation depth $d_{m}(z)$ is calculated as a function of the pressure applied to the tablet $\left(f_{n}\right)$ employing a lookup table of force matrices linearly interpolated between stored values:

$$
\begin{aligned}
& f_{n}=0.1008 e^{4.2081 f_{l},} \\
& f_{n}=0.0772 e^{3.0727 f_{l},}
\end{aligned}
$$

where $f_{l}$ is the force level reading using the getPressure() method in Android SDK and $f_{n}$ is the corresponding normal force. 


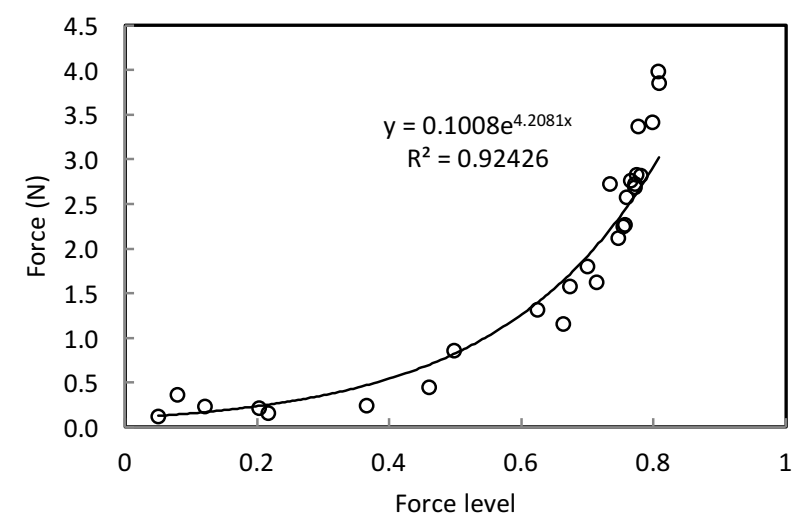

Fig. 7 Force level and force value mapping of the Samsung Note tablet (using an S-pen)



Fig. 8 Force level and force value mapping of the Motorola Xoom tablet (using a bare finger of the user).

The modification of the avatar display ratio $R$ is realized by adding a time delay to the indenter avatar's rendering task when the indenter itself is approaching a stiffer area. If the indenter passes over the stiffer area, the indenter avatar continued to follow the contact point. The delay time is determined by equation (6):

$$
\begin{aligned}
& t_{d}=\Delta f_{t} \cdot m, \\
& \Delta f_{t}=f_{t}-f_{t l},
\end{aligned}
$$

where the value of the tangent reaction force $f_{t}$ is acquired from the reaction force matrices formed during the rolling indentation; $f_{t l}$ is the tangent reaction force value at the last avatar position; $\Delta f_{t}$ is the reaction force difference; and $m$ is a scalar value set to be 500 in this study. The calculated time delay is then added to the program frame interval time. Fig. 9 shows the user interfaces of the pseudo-haptic soft object stiffness simulation using the two tablet computers. 




(a)



(b)

Fig. 9 Pseudo-haptic soft object stiffness simulation using tablet computers: (a) Samsung Galaxy Note 10.1 (using an S-pen) and (b) Motorola Xoom (using a bare finger of the user).

\section{Evaluation experiments}

\subsection{Measuring soft object stiffness distribution}

The stiffness distribution matrices used in the pseudo-haptic soft object stiffness simulation corresponded to a $120 \times 120 \times 25 \mathrm{~mm}^{3}$ silicone block with three spherical nodules embedded inside (see Fig. 10). The phantom was manufactured using RTV6166 (TECHSIL Limited, UK) (A : B=4 : 6). The nodules were made from STAEDTLER Mars plastic 52650 (47-50 ShoreA) rubber. The diameters of the spherical nodules used were $10 \mathrm{~mm}, 8 \mathrm{~mm}$, and $6 \mathrm{~mm}$, respectively. All of the nodules were buried at a depth of $6 \mathrm{~mm}$; this distance was measured from the top of the each nodule to the silicone surface. To obtain a rolling/sliding stiffness map [6], [23]-[25], 59 straight-line trajectories (121 mm long and parallel to the $x$-axis with a interval of $4 \mathrm{~mm}$ between trajectories along the $y$-axis) were defined. A robot arm (Mitsubishi RV-6SL 6-DOFrobotic manipulator with a positioning accuracy of 0.01 $\mathrm{mm}$ ) was programmed to move attached the rolling indentation probe along these 59 straight-line trajectories at a speed of $30 \mathrm{~mm} / \mathrm{s}$ with a constant rolling indentation depth. The normal and tangent components of reaction forces were recorded at a sampling rate of $100 \mathrm{~Hz}$. This experiment was repeated for a range of rolling indentation depths between $2 \mathrm{~mm}$ and $7 \mathrm{~mm}$ with intervals of $1 \mathrm{~mm}$. Six $159 \times 59$ normal and tangent force distribution matrices were created, which allowed us to obtain a stiffness distribution map for the whole silicone block surface. Here, force distribution matrices were recorded using a Nano17-ATI force sensor. Subsequently, this information was used in the algorithm of pseudo-haptic feedback.

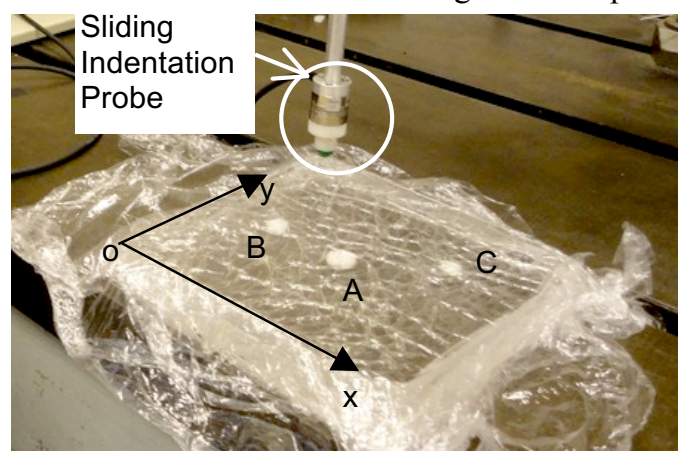

Fig. 10 The silicone block and the locations of the three embedded hard inclusions (A,B,C).

\subsection{Test Protocol}

In order to validate our pseudo-haptic soft object stiffness simulation approach, a series of experiments were conducted employing the aforementioned three types of 2-DOF force-sensitive haptic surfaces.

Pseudo-haptic feedback relies on visual display, so people with uncorrectable visual impairment cannot participant in the test. 
Here, two groups of participants, those who have normal vision and those with corrected vision, participated in an empirical study. The first group (Group I) consisted of twenty participants of which nineteen right-handed, one left-handed, one with palpation experience, and all with an engineering background while the second group (Group II) consisted of twenty participants, all right-handed, with an engineering background but no palpation experience (see Table I).

Group I conducted the tests of manual hard inclusions detection using direct hand touch (Manual I) and the pseudo-haptic soft object stiffness simulation using a force-sensitive touchpad (Touchpad). Group II participated in the tests of manual hard inclusions detection using direct hand touch (Manual II) and the pseudo-haptic soft object stiffness simulation using a tablet with an S-pen input (S-pen) and a tablet with a bare finger input (Bare finger). Prior to the start of each test, participants were also asked to do a practice run with known tumor locations. Subsequently, they were requested to manipulate the input device, palpate the virtual soft object and observe the change of the ratio between the indenter avatar displacement and the input device displacement. These participants were also asked to report the positions of hard inclusions, where they detected them. Throughout the whole programme of tests, we recorded the correctly and wrongly identified hard inclusions. It is worth mentioning that during all the aforementioned tests, the same stiffness map applied (one silicone/ nodule block was used), but the orientation of the stiffness map was different from test to test: so the participants would not learn the nodules' locations from the earlier tests. For each participant, the tests were conducted in a pseudo random order. During the manual hard inclusions detection test, the transparent silicone block surface was covered by a black plastic sheet to prevent the locations of the hard inclusions from being visible to the user.

Table 1.

\begin{tabular}{|c|c|c|}
\hline Item & Group I & Group II \\
\hline Age range & $23-42$ & $20-30$ \\
\hline Gender & oq: $6 ;$ ภ̊: 14 & o: $7 ;$ ภ̊: 13 \\
\hline Handedness & $\mathrm{R}: 19 ; \mathrm{L}: 1$ & $\mathrm{R}: 20 ; \mathrm{L}: 0$ \\
\hline Palpation experience & 1 & 0 \\
\hline Engineering background & 20 & 20 \\
\hline VR simulator & 0 & 0 \\
\hline
\end{tabular}

\section{$4 \quad$ Results and Discussion}

\subsection{Results}

The overall $\mathrm{Se}$ of each test is shown in Fig. 11. The Sensitivity $(S e)$, which relates to the test's ability to identify positive results of the presence of hard nodules, is defined as sum over all the $n$ trials of the True Positives $(T P)$ divided by the actual number of hard inclusions or the sum of TP and False Negatives $(F N)$, namely:

$$
S e=\sum_{i=1}^{n} T P_{i} / \sum_{i=1}^{n}\left(T P_{i}+F N_{i}\right) .
$$

Wilson score intervals, which have good properties even for a small number of trials (less than 30) and/or an extreme probability, were calculated for sensitivity at a $95 \%$ confidence level using the following formula:

$$
\frac{1}{1+\frac{z^{2}}{n}}\left[\hat{p}+\frac{z^{2}}{2 n} \pm z \sqrt{\frac{\hat{p}(1-\hat{p})}{n}+\frac{z^{2}}{4 n^{2}}}\right] \text {, }
$$

where $\hat{p}$ is the proportion of successes estimated from the statistical sample; $z$ is the $1-\alpha / 2$ percentile of a standard normal distribution; $\alpha$ is the error percentile and $n$ is the sample size. Here, since the confidence level is $95 \%$, the error $\alpha$ is $5 \%$.

The best $S e$ was achieved with the tablet and S-pen (91.7\%). The $S e$ of each pairs of tests was compared. It was conducted by comparing the observed probabilities $\left(p_{1}\right.$ and $\left.p_{2}\right)$ with a combined interval $(C I)$, which was calculated by the following formula:

$$
C I=\sqrt{\left(P_{1}-p_{1}\right)^{2}+\left(P_{2}-p_{2}\right)^{2}},
$$

where $P_{1}$ is the upper bound of $p_{1}$ and $P_{2}$ is the lower bound of $p_{2}$ if $p_{1}<p_{2}$. If $\left|p_{1}-p_{2}\right|>C I$, there is a significant difference between the two tests. Table II shows the test results. In comparison with Group II, Group I had a higher $\mathrm{Se}(88.3 \%$ vs. $73.3 \%)$. However, the two methods had no significant difference in $\operatorname{Se}(C I=0.148, \Delta p=0.100)$. This proved that there was no significant difference in the touch perception abilities of these two groups. One can notice that both the Se of using tablet and S-pen, and tablet and bare finger were significantly higher than using a touchpad. Thus, direct touch and immersive illusion was proven to be superior to when visual and haptic information did not present at the same point of interaction. In comparison with using a tablet with a bare finger input, using a tablet with an S-pen input had higher $S e(91.7 \%$ vs. 85\%). However, the difference was not significant $(C I=0.157, \Delta p=$ $0.067)$.

Fig. 12 compares the detection sensitivity of nodules A, B and C through the different pseudo-haptic feedback methods. Using 
the touchpad, the $\mathrm{Se}$ of nodule B was the highest (75\%), followed by nodule A (65\%); it was interesting to note that nodule B had a higher $\mathrm{Se}$ despite being smaller than nodule A. The smallest tumor $\mathrm{C}$ had a low $\mathrm{Se}$ of $15 \%$. Using the tablet with an S-pen input, the $\mathrm{Se}$ of both nodule A and B were $100 \%$. The smallest tumor, C, had a $S e$ of $75 \%$. Using the tablet with a bare finger input, both the $\mathrm{Se}$ of nodule A and B were $95 \%$. The smallest tumor C had a Se of $65 \%$.

Fig. 13 presents the Positive Predictive Values $(P P V)$, or precision rates, which are defined as sum over all the $n$ trials of the $T P$ divided by the test outcome positive or the sum of $T P$ and False Positives $(F P)$ - participants claim there is a hard nodule when there is no one, namely:

$$
P P V=\sum_{i=1}^{n} T P_{i} / \sum_{i=1}^{n}\left(T P_{i}+F P_{i}\right) .
$$

The best $P P V$ was achieved by using the tablet with an S-pen input (100\% with $95 \%$ confidential interval: $93.5-100 \%)$ followed by using the tablet with a bare finger input $(100 \%, 93.0-100 \%))$. Using the touchpad had the lowest $P P V(86.1 \%: 71.3-97.1 \%)$. The $P P V$ using the touchpad $(C I=0.139, \Delta p=0.167 ; C I=0.142, \Delta p=0.167 ; C I=0.142, \Delta p=0.167 ; C I=0.145, \Delta p=0.167)$ was significantly lower than the other four tests.

\subsection{Discussion}

Using a bare finger to provide the indentation and sliding input makes the contact more natural than using a pen. However, we noticed that the Se of using a tablet and a bare finger was lower than that of using a tablet and an S-pen. One reason for this is that the fingertips are larger than the S-pen tip. Touching the screen with a bare finger can sometimes obstruct the vision of the user during the experiments. Another reason can be the saturation problem of the pressure level measurement of this device, namely, using the contact surface area to estimate the contact force is not reliable when the contact area reaches its maximum while the contact force is still increasing. Hence, a better pressure-sensitive touch screen for bare finger interaction is required.

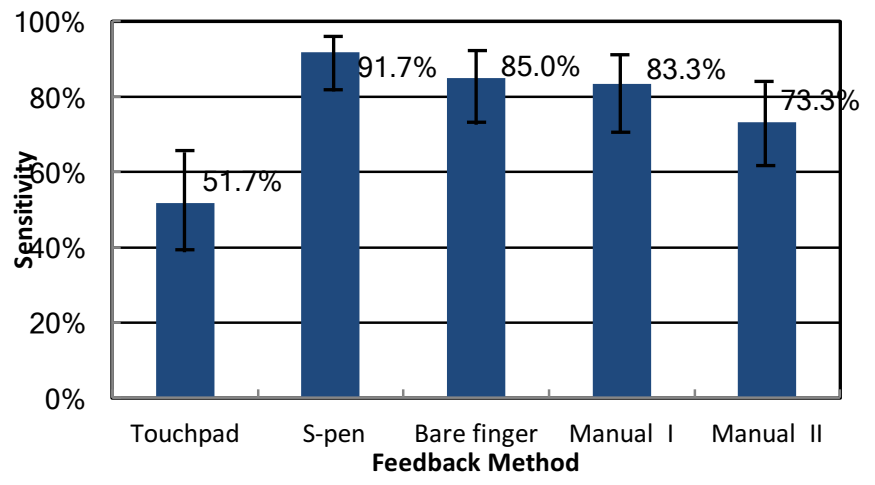

Fig. 11 Overall nodule detection sensitivities with Wilson score intervals at a $95 \%$ confidence level. Touchpad represents the pseudo-haptic soft object stiffness simulation using a touchpad; S-pen represents the experiment using a tablet with a motion and force input from an S-pen; Bare finger represents the experiment using a tablet with a motion and force input from a bare finger of the user; Manual I represents hard inclusions detection using hand-soft object interaction conducted by Group I; Manual II represents hard inclusions detection using hand-soft object interaction conducted by Group II.

Table 2.

Comparsion of sensitivities

\begin{tabular}{|c|c|c|c|}
\hline$x^{2}$ & $\begin{array}{c}\text { Combined } \\
\text { interval } \\
(C I)\end{array}$ & $\begin{array}{c}\text { Probability difference } \\
(\Delta p)\end{array}$ & Significance? \\
\hline Manual II \& Manual I & 0.148 & 0.100 & No \\
\hline Manual I \&Touchpad & 0.167 & 0.316 & Yes \\
\hline S-pen \& Bare finger & 0.157 & 0.067 & No \\
\hline S-pen \& Touchpad & 0.156 & 0.400 & Yes \\
\hline S-pen \& Manual II & 0.136 & 0.184 & Yes \\
\hline Bare finger \& Touchpad & 0.165 & 0.333 & Yes \\
\hline Bare finger \& Manual II & 0.146 & 0.117 & No \\
\hline
\end{tabular}




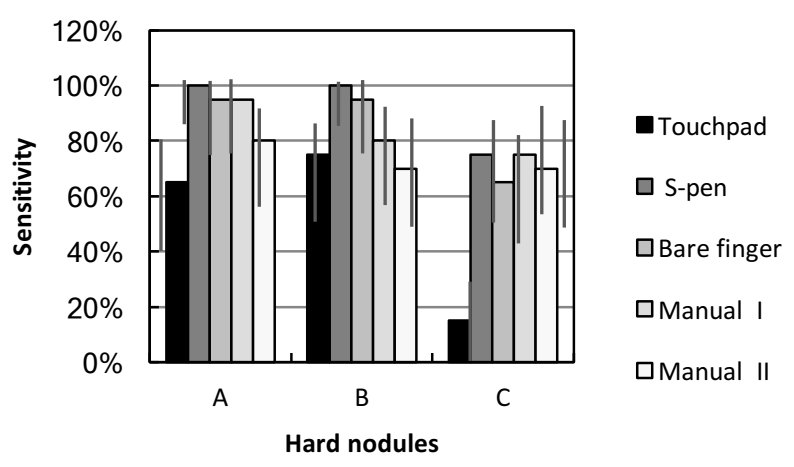

Fig. 12 Nodule detection sensitivities of nodule A, B, and C with Wilson score intervals at a $95 \%$ confidence level.



Fig. 13 Positive predictive values with Wilson score intervals at a 95\% confidence level.

\section{Conclusions}

The performance of the proposed pseudo-haptics rigid tool / soft object interaction technique has been assessed using a force-sensitive touchpad with a pen input, a tablet computer with an S-pen input, and a tablet computer with a user's bare finger input. Hard inclusions were detected more effectively on tablet computers. Sensitivity and positive predictive value were lower when the force-sensitive touchpad was used as visual and haptic information was presented at different points of interaction. Notably, the detection of hard inclusions done by applying direct touch or immersive illusion on tablet computers was comparable to detection performed with the use of hand / soft object interaction.

The proposed technique is an effective and low-cost alternative to conventional haptic devices and impresses with its performance in the detection of hard inclusions which rivals detection done via hand / soft object interaction. Potential applications include remote medical palpation. Using archived stiffness distribution information (acquired for instance via the rolling/sliding indentation method) and pseudo-haptic soft object stiffness simulation, a surgeon could examine a patient without actual skin-to-skin contact. In addition, with the video gaming community always in the look for more realistic experiences, our proposed technique is sure to find many applications in gaming too.

\section{Acknowledgement}

The work described in this paper was partially funded by the National Institute for Health Research (NIHR) Biomedical Research Centre based at Guy's and St Thomas' NHS Foundation Trust and King's College London, the European Commission's Seventh Framework Programme under grant agreement 287728 in the framework of EU project STIFF-FLOP, and the China Scholarship Council. The views expressed are those of the authors and not necessarily those of the NHS, the NIHR or the Department of Health.

\section{References}

[1] A. Lecuyer, S. Coquillart, A. Kheddar, P. Richard, and P. Coiffet, "Pseudo-haptic feedback: can isometric input devices simulate force feedback?," in Proceedings IEEE Virtual Reality 2000 (Cat. No.00CB37048), 2000, pp. 83-90.

[2] A. Lecuyer, J.-M. Burkhardt, S. Coquillart, and P. Coiffet, “'Boundary of illusion': an experiment of sensory integration with a pseudo-haptic system," Proc. IEEE Virtual Real. 2001, pp. 115-122, 2001.

[3] T. Kimura and T. Nojima, "Pseudo-haptic feedback on softness induced by grasping motion," in EuroHaptics 2012, 2012, pp. 202-205.

[4] L. Dominjon, A. Lecuyer, J. Burkhardt, P. Richard, and S. Richir, "Influence of control/display ratio on the perception of mass of manipulated objects in virtual environments," IEEE Proceedings. VR 2005. Virtual Reality, 2005., vol. 2005, pp. 19-25, 2005.

[5] A. Lécuyer, J.-M. Burkhardt, and C.-H. Tan, "A study of the modification of the speed and size of the cursor for simulating pseudo-haptic bumps and holes," ACM Trans. Appl. Percept., vol. 5, no. 3, pp. 1-21, Aug. 2008.

[6] J. Zirjakova, "Prostate post-surgical 3D imaging and data analysis," 2011

[7] R. Balaniuk, "A differential method for the haptic rendering of deformable objects," Proc. ACM Symp. Virtual Real. Softw. Technol. - VRST '06, p. $297,2006$.

[8] K. Salisbury, F. Conti, and F. Barbagli, "Haptic rendering: introductory concepts.," IEEE Comput. Graph. Appl., vol. 24, no. 2, pp. 24-32, 2004. 
[9] SensAble Technologies, "SensAble Technologies," 2012. [Online]. Available: http://www.sensable.com.

[10] Force Dimension, "Force Dimension," 2012. [Online]. Available: http://www.forcedimension.com.

[11] Inc. Novint Technologies, "Novint Technologies," 2012. [Online]. Available: http://home.novint.com.

[12] H. Kawasaki, J. Takai, Y. Tanaka, C. Mrad, and T. Mouri, "Control of multi-fingered haptic interface opposite to human hand," in Proceedings 2003 IEEERSJ International Conference on Intelligent Robots and Systems IROS 2003, 2003, vol. 3, no. October, pp. 2707-2712.

[13] V. Daniulaitis and M. O. Alhalabi, "Medical Palpation of Deformable Tissue using Physics-Based Model for Haptic Interface RObot ( HIRO )," in Information Systems, 2004, pp. 3907-3911.

[14] T. Endo, H. Kawasaki, T. Mouri, Y. Doi, T. Yoshida, Y. Ishigure, H. Shimomura, M. Matsumura, and K. Koketsu, "Five-fingered haptic interface robot: HIRO III," World Haptics 2009 Third Jt. EuroHaptics Conf. Symp. Haptic Interfaces Virtual Environ. Teleoperator Syst., vol. 4, no. 1, pp. 458-463, 2009.

[15] N. A. Langrana, G. Burdea, K. Lange, D. Gomez, and S. Deshpande, "Dynamic force feedback in a virtual knee palpation.," Artif. Intell. Med., vol. 6, no. 4, pp. 321-333, 1994.

[16] M. Dinsmore, N. Langrana, G. Burdea, and J. Ladeji, "Virtual Reality Training Simulation for Palpation of Subsurface Tumors," in IEEE Virtual Reality Annual International Symposium, 1997, pp. 54-60.

[17] Y. Kuroda, M. Nakao, T. Kuroda, H. Oyama, M. Komori, and T. Matsuda, "FEM-based interaction model between elastic objects for indirect palpation simulator.," Stud. Health Technol. Inform., vol. 98, pp. 183-189, 2004.

[18] A. Crossan, S. Brewster, S. Reid, and D. Mellor, “A horse ovary palpation simulator for veterinary training," Haptic human-computer Interact., pp. 157-164, 2001.

[19] R. Parkes, N. Forrest, and S. Baillie, "A mixed reality simulator for feline abdominal palpation training in veterinary medicine.," Stud. Health Technol. Inform., vol. 142, pp. 244-246, Jan. 2009.

[20] M. Nakao, T. Kuroda, M. Komori, and H. Oyama, "Evaluation and user study of haptic simulator for learning palpation in cardiovascular surgery," Int. Conf. Artif. Real. Telexistence, pp. 203-208, 2003.

[21] S. Ullrich and T. Kuhlen, "Haptic palpation for medical simulation in virtual environments.," IEEE Trans. Vis. Comput. Graph., vol. 18, no. 4, pp. 617-625, 2012.

[22] S. Khan, "Design and optimization of parallel haptic devices," 2012.

[23] H. Liu, D. P. Noonan, B. J. Challacombe, P. Dasgupta, L. D. Seneviratne, and K. Althoefer, "Rolling mechanical imaging for tissue abnormality localization during minimally invasive surgery.," IEEE Trans. Biomed. Eng., vol. 57, no. 2, pp. 404-14, Feb. 2010.

[24] H. Liu, J. Li, and Q. Poon, "Miniaturized force-indentation depth sensor for tissue abnormality identification during laparoscopic surgery," in International Conference on Robotics and Automation (ICRA), 2010, pp. 3654-3659.

[25] D. P. Noonan, H. Liu, Y. H. Zweiri, K. A. Althoefer, and L. D. Seneviratne, "A Dual-Function Wheeled Probe for Tissue Viscoelastic Property Identification during Minimally Invasive Surgery," in IEEE International Conference on Robotics and Automation, 2007, no. April, pp. 2629-2634.

[26] M. Li, L. D. Seneviratne, P. Dasgupta, and K. A. Althoefer, "Virtual palpation system," in International Conference on Intelligent Robots and Systems workshop "Learning and Interaction in Haptic Robots," 2012.

[27] M. Ridzuan, Y. Makino, and K. Takemura, "Direct touch haptic display using immersive illusion with interactive virtual finger," EuroHaptics 2012, pp. 432-444, 2012.

[28] A. Withana, Y. Makino, M. Kondo, M. Sugimoto, G. Kakehi, and M. Inami, "ImpAct: Immersive haptic stylus to enable direct touch and manipulation for surface computing," Comput. Entertain., vol. 8, no. 2, p. 1, Dec. 2010. 